\title{
Article
}

\section{Translucency of Graphene to van der Waals Forces Applies to Atoms/Molecules with Different Polar Character.}

Francesco Presel, Alfonso Gijón, Eduardo R. Hernández, Paolo Lacovig, Silvano Lizzit, Dario Alfè, and Alessandro Baraldi

ACS Nano, Just Accepted Manuscript • DOI: 10.1021/acsnano.9b07277 • Publication Date (Web): 07 Oct 2019

\section{Just Accepted}

"Just Accepted" manuscripts have been peer-reviewed and accepted for publication. They are posted online prior to technical editing, formatting for publication and author proofing. The American Chemical Society provides "Just Accepted" as a service to the research community to expedite the dissemination of scientific material as soon as possible after acceptance. "Just Accepted" manuscripts appear in full in PDF format accompanied by an HTML abstract. "Just Accepted" manuscripts have been fully peer reviewed, but should not be considered the official version of record. They are citable by the Digital Object Identifier (DOI®). "Just Accepted" is an optional service offered to authors. Therefore, the "Just Accepted" Web site may not include all articles that will be published in the journal. After a manuscript is technically edited and formatted, it will be removed from the "Just Accepted" Web site and published as an ASAP article. Note that technical editing may introduce minor changes to the manuscript text and/or graphics which could affect content, and all legal disclaimers and ethical guidelines that apply to the journal pertain. ACS cannot be held responsible for errors or consequences arising from the use of information contained in these "Just Accepted" manuscripts. 


\title{
Translucency of Graphene to van der Waals
} Forces Applies to Atoms/Molecules with Different Polar Character.

\author{
Francesco Presel, ${ }^{\dagger, @ ~ A l f o n s o ~ G i j o ́ n, ~}, \stackrel{\ddagger}{ }$ Eduardo R. Hernández, ${ }^{\ddagger}$ Paolo Lacovig,, \\ Silvano Lizzit, "Dario Alfe, $, \AA, \|, \perp$ and Alessandro Baraldi ${ }^{*},+, \pi, \#$ \\ $\dagger$ Physics Department, University of Trieste, Via Valerio 2, 34127 Trieste, Italy \\ $\ddagger$ Instituto de Ciencia de Materiales de Madrid - ICMM-CSIC), Campus de Cantoblanco, \\ 28049 Madrid, Spain \\ \Elettra-Sincrotrone Trieste S.C.p.A., Strada Statale 14 Km 163.5, 34149 Trieste, Italy \\ $\S$ Department of Earth Sciences, Department of Physics and Astronomy, TYC@UCL \\ ||London Centre for Nanotechnology, University College London, Gower Street, London \\ WC1E 6BT, United Kingdom \\ $\perp$ Dipartimento di Fisica Ettore Pancini, Università di Napoli Federico II, Monte S. \\ Angelo, 80126 Napoli, Italy \\ \#IOM-CNR, Laboratorio TASC, AREA Science Park, S.S. 14 km 163.5, 34149 Trieste, \\ Italy \\ @ Current address: DTU Physics, Technical University of Denmark, 2800 Kgs. Lyngby, \\ Denmark \\ E-mail: alessandro.baraldi@elettra.eu
}

Phone: +390403758719 


\section{Abstract}

Graphene has been proposed to be either fully transparent to van der Waals interactions to the extent of allowing to switch between hydrophobic and hydrophilic behaviour, or partially transparent (translucent), yet there has been considerable debate on this topic, which is still ongoing. In a combined experimental and theoretical study we investigate the effects of different metal substrates on the adsorption energy of atomic (argon) and molecular (carbon monoxide) adsorbates on high-quality epitaxial graphene. We demonstrate that while the adsorption energy is certainly affected by the chemical composition of the supporting substrate and by the corrugation of the carbon lattice, the van der Waals interactions between adsorbates and the metal surfaces are partially screened by graphene. Our results indicate that the concept of graphene translucency, already introduced in the case of water droplets, is found to hold more generally also in the case of single polar molecules and atoms, which are apolar.

\section{Keywords}

graphene, molecules, atoms, translucency, van der Waals, adsorption energy, physisorption

Besides its interest at a fundamental level, the interaction of graphene (Gr) with atoms and molecules attracts a great deal of attention among the scientific community because of its possible application as a metal-free active phase in catalysis ${ }^{1 / 3}$ or as a co-catalyst in photocatalysis, $\stackrel{45]}{a}$ as well as in gas sensing. ${ }^{6}$ However, in such applications Gr will be used not in its free-standing state, but rather supported on a substrate, which is known to modify its physical and chemical properties. ${ }^{7}$ The interaction with different substrates can be rather 
variable, due to the different degree of substrate-Gr charge redistribution, which can modify its doping level and the hybridization of the carbon atoms. ${ }^{8}$

In this respect, there is an on-going debate in the scientific community concerning the role of the supporting substrate on the adsorption properties of atoms and molecules on Gr. Up to now, this debate has mainly focussed on the case of water, as this system, besides providing fundamental understanding, plays a vital role for the possibility of biological applications of graphene. ${ }^{9[10}$ By combining contact angle measurements with molecular dynamics simulations, Rafiee et al. suggested that graphene could be fully transparent to van der Waals (vdW) interactions, 11 a result that is ascribed to the extreme thinness of Gr. This is an interesting result as vdW interactions are known to be generally non-additive, i.e. the total interaction of one adsorbate with two adjacent materials is not the sum of the interactions with each of them. In contrast, Shih et al., on the basis of classical theory of vdW interactions, reported that the transparency is only partial - i.e. a layer of Gr does reduce the interaction with the underlying substrate, yet only to about $30 \%$ of its original value, and would then be more appropriately described as translucent, $\frac{12}{12}$ and other studies have denied any effect of the substrate on the graphene-water interaction. ${ }^{13}$ In addition, two possible mechanisms have been identified by which the substrate below Gr can contribute to the adsorption energy of molecules. The first is a purely dispersive, direct interaction of the molecules with the substrate below Gr, i.e., a real translucency to vdW forces. The other is an indirect effect on the interaction between the molecules and Gr due to the modifications induced by the substrate on the electronic properties of $\mathrm{Gr}$, which despite appearing as a form of "translucency", is more correctly described as a modification of the adsorption properties

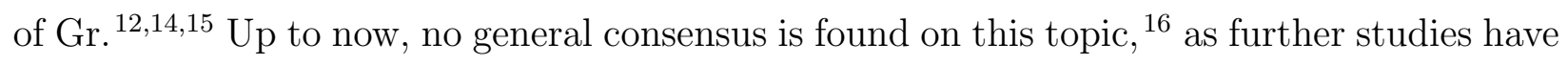
even recently lead to incompatible results, either upholding $\frac{14 \mid 17}{\text { or rejecting }}{ }^{15[18 \mid 19}$ this real translucency, to various degrees. $\frac{1020}{2 n}$ particular, in the most recent literature, it has been reported ${ }^{15}$ that the hydrophilicity of graphene can be intimately connected to the position of its Fermi level, which affects the interaction between graphene and water molecules. This 
was verified by applying a voltage between $-100 \mathrm{~V}$ and $+100 \mathrm{~V}$ to graphene, showing that this doping effect is capable of modifying the adsorption configuration of water molecules by adjusting to the doping state of graphene, but the reason does not appear to be the vdW interaction. Similar effects of tunable wettability were obtained also by changing the subsurface metal, and explained in terms of inducing a different graphene doping. 19 These papers, therefore, suggest that the effect of the substrate is indirect, and not a real translucency. On the other hand, a combined water contact angle and Density Functional Theory (DFT) study performed on Gr supported on different substrates claims that Gr is translucent to both polar and dispersive interactions alike. $\stackrel{10}{10}$ this work, we shed light and deconvolute the role of these two proposed mechanism and identify the dominant one in determining the interaction of atoms/molecules with supported Gr.

Chemically inert materials such as graphene, if proven transparent to electronic transfer and interaction, could be very promising for the development of passivating films allowing to employ electrocatalytically active materials in harsh environments while protecting them from corrosion. 21

The intriguing role of the underlying surface in modifying the Gr-adsorbate interaction is not limited to the specific case of water molecular adsorption: also in the case of carbon monoxide, adsorption on supported Gr shows interesting characteristics. Experimental ${ }^{22}$ and theoretical ${ }^{23}$ results have shown how the adsorption energy of CO on Gr strongly interacting with a $\mathrm{Ni}(111)$ surface is significantly larger than in the case of $\mathrm{CO}$ on free-standing $\mathrm{Gr}$; because of this, this system has been hailed as a promising metal-free catalyst.24

Beyond the case of adsorbates, this effect also holds for extended two-dimensional layers grown above Gr. For example, very recent results of Kong et al. $\frac{25}{20 m o n s t r a t e ~ t h a t ~ C o u l o m b ~}$ interactions can be transmitted through graphene to the point of inducing the in-registry growth of the same material above and below graphene when the material used has a sufficiently strong polar character (e.g. GaAs, GaN, or LiF); however, the same does not happen for covalently bonded materials (e.g. Si, Ge), due to the short range of the covalent bonding 
interaction.

However, it is very important to remind that the adsorption energy of atoms and molecules on Gr is known to be affected by several contributions: the first is the presence of defects, such as vacancies and domain boundaries, where the local coordination of the Gr atoms and therefore their reactivity towards adsorbates is different, $\frac{26}{26}$ and contaminants, which are known to modify the degree of Gr doping. In addition the adsorbate-graphene interaction can be influenced by the degree of adhesion of graphene with the underlying substrate, such as the one introduced by the physical transfer process from the growth substrate, which can results in corrugation, wrinkles and non-uniformity of the sample. However, also in the case of graphene prepared on cyrstalline surfaces, multiple phases, characterized by different translational or rotational symmetry, can coexist, leading to a changeable degree of interaction with the substrate in different regions. $\frac{27}{27}$ Finally, the superposition of the graphene unit cell to that of the substrate can induce a moirè-driven buckling of Gr, leading to a variable Gr-substrate distance on the nanometre scale. ${ }^{8}$ This variable distance, besides affecting the electronic structure of Gr, could significantly influence the vdW interaction, due to its long-range dependence on the distance of the adsorbates from the substrate: $\frac{[12}{10}$ for example, it has been shown that hydrogen preferentially adsorbs on the convex regions of buckled graphene/SiC(0001). $\stackrel{28}{ }$

All these factors can affect the molecular adsorption properties of Gr at the local scale. This makes their experimental study challenging as the adsorption energy is typically probed by measuring the wetting angle of water droplets, i.e. a macroscopic quantity, which is known not to be a precise approach to probe the transparency of graphene. $\frac{25}{25}$

Defect-free $\mathrm{Gr}$ is chemically rather inert; it therefore follows that the interaction with adsorbates can be expected to be dominated by dispersion forces, although there is some evidence of hybridization with some adsorbates such as ammonia. ${ }^{29}$ This poses a significant challenge for the theoretical study of Gr-adsorbate interactions; indeed dispersion forces are difficult to account for within density-functional theory calculations, the methodology of 
choice when addressing systems as large as the ones considered here. Several approaches exist to do so, but their relative merits in terms of accuracy are still to be established, and need to be tested in each particular case.

To reduce and control the degrees of freedom affecting the adsorption dynamics of atoms/molecules on Gr, in order to understand and quantify their different effects on the atomic and molecular adsorption on Gr, we have employed a combined experimental and theoretical approach. In particular, our strategy is based on the comparison of the adsorption energy of $\mathrm{CO}$ molecules and Ar atoms deposited in different concentrations on two different interfaces, namely $\operatorname{Gr} / \operatorname{Ir}(111)$ - where Gr is considered almost fully decoupled from this substrate $\stackrel{30 / 31}{-}$ - and $\mathrm{Gr} / \mathrm{Co} / \operatorname{Ir}(111)$. In addition, in our experiments, we obtained a direct measurement of the adsorption energy of our species by employing the Temperature Programmed-XPS (TP-XPS) technique. ${ }^{32}$ This technique, described in detail in the Methods section, allows to probe variations in the adsorption sites, configurations and adsorbate electronic properties as a function of the temperature and residual molecular/atomic coverage, and therefore to account for any contribution due to local defects.

The great advantage of our approach is that the intercalation of cobalt atoms below an extended and high-quality Gr monolayer epitaxially grown by means of chemical vapour deposition on Ir results in the formation of a corrugated structure with regions of the carbon network close to (about $1.88 \AA$ ) and far from (about $3.15 \AA$ ) the metal substrate, while preserving the orientation and the lattice mismatch of $\mathrm{Gr}$ with respect to the substrate underneath and the resulting moirè, without affecting the defects density significantly. This strategy allows us to not only determine the adsorption energy at different adsorbatemetal distances, but also to probe the effects of the graphene corrugation.

The most important results of our study show clearly that, for the very different systems we studied, regardless of substrate composition, adsorbate-metal distance and specific adsorption sites (convex or concave regions of the carbon lattice), graphene allows about $50 \%$ of the vdW interaction to pass through. Our findings show that dispersion interactions, 
which are also of a longer range than covalent bonding, can be transmitted, although partially screened, through a graphene monolayer, thus suggesting that the concept of graphene translucency, which has been put forward in the case of water, can be applied also in the case of single molecules and atoms, which show a net dipole moment or not.

\section{Results and Discussion}

\section{Experimental Results}

\section{Carbon Monoxide Desorption from Graphene/Ir(111)}

The adsorption of Carbon Monoxide, which is reported in detail in the Supporting Information, was performed at $38 \mathrm{~K}$ : this temperature was chosen as it is the highest at which $\mathrm{CO}$ can adsorb, in order to ensure that the molecules have some mobility to diffuse to their equilibrium adsorption configuration, and is high enough to avoid a multilayer formation, which takes place below $30 \mathrm{~K}$. The photoemission spectrum of adsorbed CO shows a single C 1s component (V), initially centered at $289.9 \mathrm{eV}$ and shifted by $5.8 \mathrm{eV}$ with respect to the $\mathrm{C} 1 \mathrm{~s}$ component of graphene $(284.12 \mathrm{eV})$, for the whole coverage range we investigated (see Fig. 1(a), which shows selected spectra, corresponding to a coverage of 0.08 ML (bottom) and 0.30 ML (top)). The photoemission peak shows a slightly asymmetric line shape, which is due to the vibrational fine structure of the molecule, as explained in the Supporting Information.

TP-XPS measurements were then taken for different exposures of CO on $\mathrm{Gr} / \operatorname{Ir}(111)$, as shown in Fig. 1(b) for an initial CO coverage of $\theta=0.14 \mathrm{ML}$ : the $\mathrm{C}$ 1s photoemission intensity is plotted as a function of the annealing temperature, using a grey-scale density plot ranging from low (white) to high intensities (black). The coverage evolution as a function of temperature during this experiment is shown in Fig. 1(c), together with the curves obtained for other different initial coverages. 

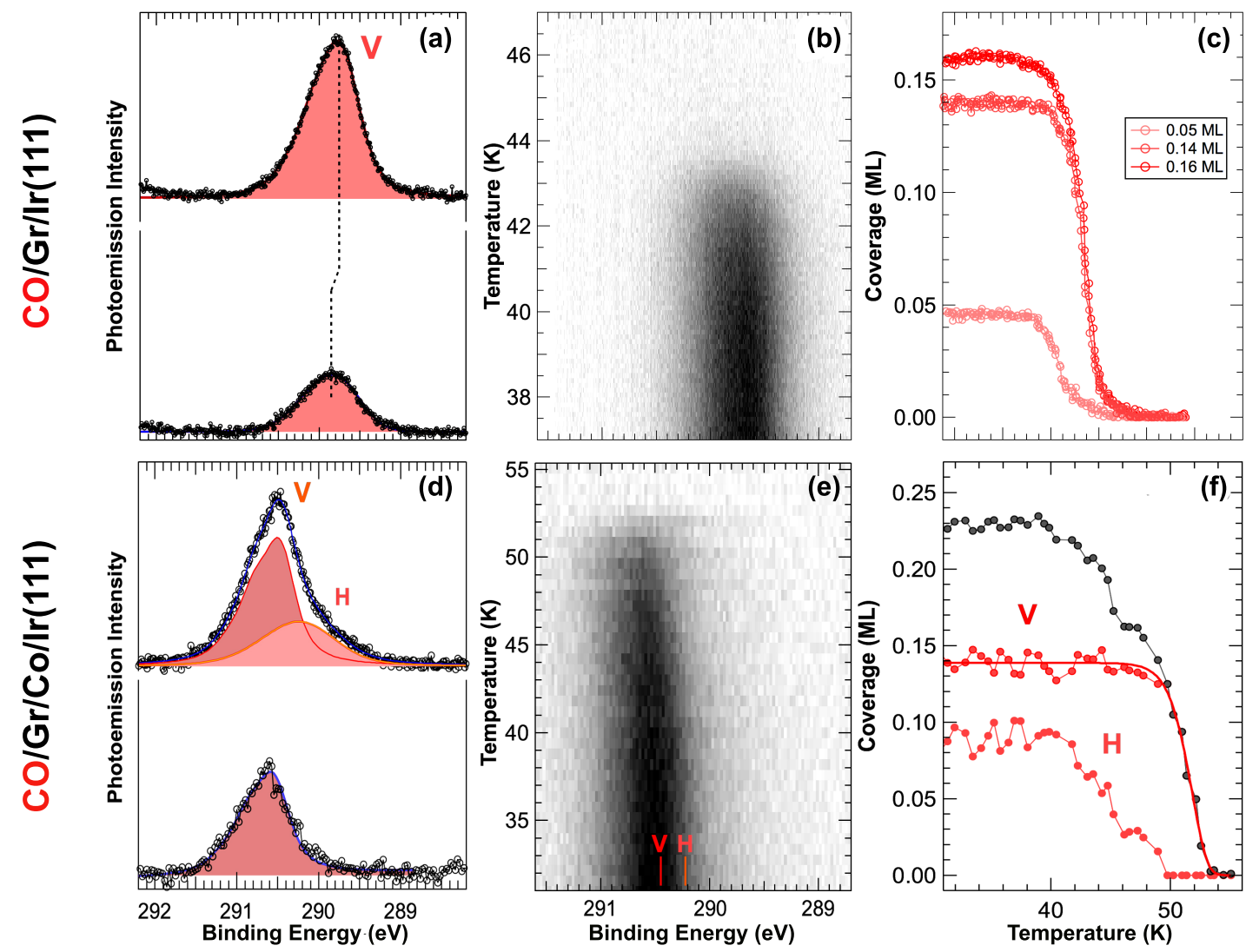

Figure 1: CO desorption from $\mathrm{Gr} / \mathrm{Ir}(111)$. (a) C1s spectra corresponding to different $\mathrm{CO}$ initial coverages $\left(\theta_{C O}=0.08 \mathrm{ML}\right.$ (bottom) and 0.30 ML (top)), measured prior to the temperature ramp. (b) TP-XPS C 1s core level spectra showing its evolution during thermal desorption of CO from $\mathrm{Gr} / \operatorname{Ir}(111)$. (c) Comparison of $\mathrm{CO}$ coverage evolution as a function of temperature for selected $\mathrm{CO}$ initial coverages. $\mathrm{CO}$ desorption from $\mathrm{Gr} / \mathrm{Co} / \operatorname{Ir}(111)$ (d) Selected spectra of the uptake corresponding to a coverage of 0.08 ML (bottom) and to saturation (top). (e) TP-XPS spectra acquired during a linear temperature ramp (the photoemission intensity is in gray scale), and (f) temperature evolution of $\mathrm{V}$ (valley) and $\mathrm{H}$ (hills) $\mathrm{C}$ 1s photoemission components of a $0.23 \mathrm{ML}$ CO dose as a function of temperature.

Each desorption curve can be well described by a first order process (Fig.11(c)). However, it is evident that the temperature at which desorption takes place is not the same, but moves towards higher temperatures for increasing initial coverage. This cannot be described by a simple first order process, unless we assume that the desorption energy is dependent on coverage.

The desorption energy $E_{\text {des }}$ of the adsorbates (which in the case of physisorption, such as in the systems we investigated, corresponds to the adsorption energy) was obtained by fitting the desorption curves to the Arrhenius equation, as reported in detail in the Methods 
While the absolute errors associated to both $\nu$ and $E_{\text {des }}$ (see Table 1 ) are comparable to the differences we are measuring, the confidence regions of the fitted curves do not overlap (see the Supporting Information) and therefore, even though the absolute values on the fit parameters are affected by an error of about $25 \mathrm{meV}$, their differences as a function of coverage can be determined with an accuracy of $\pm 5 \mathrm{meV}$. This allows us to conclude that the desorption energy displays a significant dependence on coverage.

The value obtained for the pre-exponential $\nu$ is relatively high, as values typically used to fit the desorption curves are usually centred around $10^{13} \mathrm{~s}^{-1}$. However, it has been shown that this value can vary by several orders of magnitude depending on the system, especially when the surface is not a metal - as is the case of graphene ${ }^{\sqrt{35}} \mathrm{CO}$, besides, is characterized by a relatively high $\nu$ (around $10^{14}$ to $10^{16} \mathrm{~s}^{-1}$ ) when adsorbed on different metal surfaces. $\frac{36}{}$

The value found for the adsorption energy is a clear indication that $\mathrm{CO}$ is very weekly interacting with the substrate. The adsorption energy increases with coverage by about $20 \mathrm{meV}$, from the lowest coverage investigated, corresponding to 0.04 ML, to 0.3 ML. This dependence on coverage of the adsorption energy, which is due to inter-molecular interactions, is discussed in more detail in the Supporting Information. 


\section{Carbon Monoxide Desorption from Graphene/Cobalt/Ir(111)}

The time-resolved spectra acquired during $\mathrm{CO}$ adsorption on $\mathrm{Gr} / \mathrm{Co} / \mathrm{Ir}(111)$ at $\mathrm{T}=38 \mathrm{~K}$ are reported in the Supporting Information. Selected C 1s spectra acquired during the uptake of $\mathrm{CO}$ are shown in Figure 1(d). At low coverage (Fig. 1(d), bottom), a single component (V) is visible, which has an asymmetric shape due to the vibrational excitation in the final state, as was the case for CO/Gr/Ir (see Supporting Information). The lineshape which best fits the low coverage data has a Lorentzian FWHM $L=310 \pm 20 \mathrm{meV}$ and a Gaussian FWHM $G=240 \pm 20 \mathrm{meV}$, not significantly different from the Gr/Ir case.

The $\mathrm{C}$ 1s spectrum of a saturated CO monolayer is shown in Fig. 1(d) (top). This spectrum can no longer be fitted with just a single component, but a second one $(\mathrm{H})$ has to be included at lower BE. Component $\mathrm{V}$ presents the same lineshape as in the low coverage spectrum. Component $\mathrm{H}$ can be fitted with the same vibrational quantum and FranckCondon factor as component $\mathrm{V}$, however the lineshape is characterized by a larger Gaussian broadening $G=0.76 \pm 0.12 \mathrm{meV}$, indicating a higher degree of disorder in the system. The $\mathrm{BE}$ of the $\mathrm{V}$ component in the $\mathrm{C} 1 \mathrm{~s}$ spectrum of $\mathrm{CO}$ at saturation coverage is $290.47 \mathrm{eV}$, which is significantly larger than for $\mathrm{CO} / \mathrm{Gr} / \operatorname{Ir}(111)(289.74 \mathrm{eV})$, by about $730 \mathrm{meV}$. The BE of component $\mathrm{H}$ is $290.12 \mathrm{eV}$, lower than $\mathrm{V}$ by about $350 \mathrm{meV}$. Still, both components $\mathrm{V}$ and $\mathrm{H}$ lie at a higher $\mathrm{BE}$ than those of $\mathrm{CO} / \mathrm{Gr} / \mathrm{Ir}(111)$. This $\mathrm{C} 1 \mathrm{~s}$ BE shift is actually similar to that of the $\mathrm{C} 1 \mathrm{~s}$ core level of $\mathrm{Gr}$, which displays two photoemission components when it is supported on $\mathrm{Co} / \operatorname{Ir}(111)$, both at higher BE with respect to $\mathrm{Gr} / \operatorname{Ir}(111) .34$ We can therefore attribute the different $\mathrm{C}$ 1s BE of the $\mathrm{CO}$ molecules in the two systems to the fact that they are influenced by the doping or the different geometries of the underlying $\mathrm{Gr}$ layer. At saturation, the photoemission intensity of component $\mathrm{V}$ is about 1.6 times that of component $\mathrm{H}$, with the $\mathrm{H}$ coverage $(0.095 \mathrm{ML})$ which corresponds to $39 \%$ of the overall $\mathrm{CO}$ populations.

Also for the Gr/Co/Ir(111) interface, TP-XPS measurements were taken for different initial coverages of CO. Fig. 1(e) shows a selected TP-XPS experiment, corresponding to an 
The fit of the desorption curves of component $\mathrm{V}$ yields a pre-exponential factor $\nu=10^{17 \pm 2} \mathrm{~s}^{-1}$, the same which was found for $\mathrm{CO} / \mathrm{Gr} / \mathrm{Ir}$, yet the adsorption energy in this system is higher, as reported in Table 2.

This comparison shows that the adsorption energy of $\mathrm{CO}$ on $\mathrm{Gr}$, at least up to a coverage of about $0.15 \mathrm{ML}$, is increased by about $35 \mathrm{meV}$ for the case of $\mathrm{Gr}$ on the Co-terminated $\operatorname{Ir}(111)$ surface. This substrate-induced increase in adsorption energy accounts for $20 \%$ of the total adsorption energy of $\mathrm{CO}$ in this system, therefore demonstrating that the substrate 
below Gr plays an important role in determining the overall CO interaction strength. The different behaviour which is observed at higher coverages in this system could be instead attributed to the very different geometry (different Gr corrugation) between Gr on Co/Ir(111) and on $\operatorname{Ir}(111)$, as will be shown in the next sections.

\section{Argon Desorption from Graphene/Ir(111)}
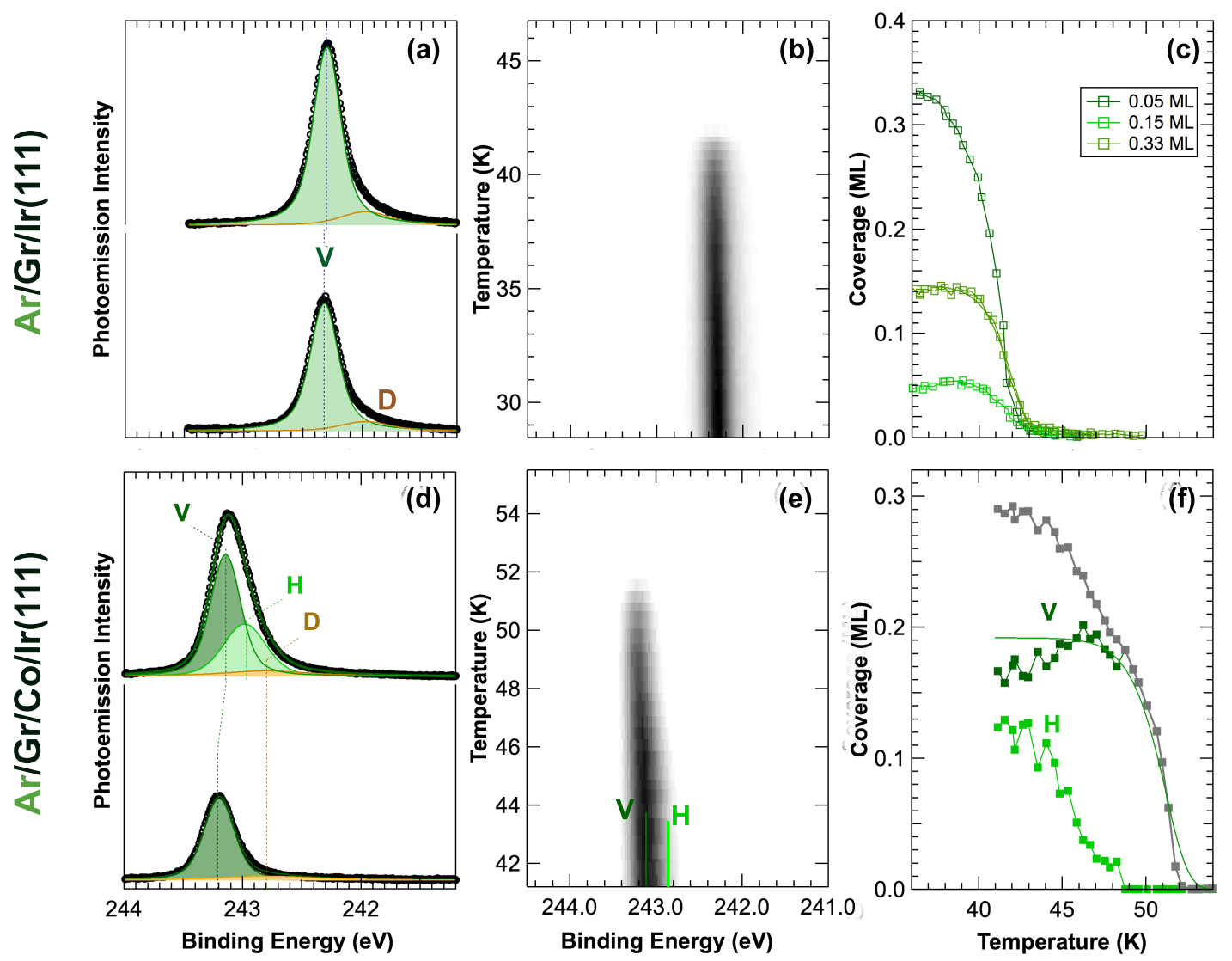

Figure 2: Ar desorption from $\operatorname{Gr} / \operatorname{Ir}(111)$. (a) Selected Ar $2 \mathrm{p}_{3 / 2}$ spectra corresponding to $\theta_{i}=0.31 \mathrm{ML}$ (bottom) and to saturation, 0.44 ML (top). (b) TP-XPS Ar 2 $\mathrm{p}_{3 / 2}$ core level spectra showing its evolution during thermal desorption of $\mathrm{Ar}$ from $\mathrm{Gr} / \mathrm{Ir}(111)$ (the photoemission intensity is in gray scale), and (c) Comparison of Ar coverage evolution as a function of temperature for different initial Ar coverages. Ar desorption from $\mathrm{Gr} / \mathrm{Co} / \mathrm{Ir}(111)$. (d) Selected $\mathrm{Ar} 2 \mathrm{p}_{3 / 2}$ spectra $(h \nu=400 \mathrm{eV})$ corresponding to a coverage of $0.21 \mathrm{ML}$ (bottom) and to saturation (top). (e) TP-XPS spectra acquired during a linear temperature ramp (the photoemission intensity is in gray scale), and (f) temperature evolution of V (valley) and $\mathrm{H}$ (hills) $\mathrm{C}$ 1s photoemission components of a 0.14 ML Ar dose as a function of temperature.

In order to probe the interaction of a different adsorbate, we studied the desorption of different coverages of Ar, which was dosed on $\mathrm{Gr} / \operatorname{Ir}(111)$ at $25 \mathrm{~K}$. Time-resolved spectra 
acquired during the uptake are reported in the Supporting Information. Selected Ar $2 \mathrm{p}_{3 / 2}$ spectra, corresponding to a coverage of $0.31 \mathrm{ML}$ and $0.44 \mathrm{ML}$, are shown in Figure 2(a). While a main component (V), at a BE of $242.32 \mathrm{eV}$, dominates the spectral distribution, with a FWHM narrower than $300 \mathrm{meV}$, a broader low intensity peak (D), centred at about $242 \mathrm{eV}$, with a FWHM of about $500 \mathrm{meV}$, has to be included to correctly fit the spectrum. After the initial growth the latter component remains constant throughout the whole uptake and its spectral weight is significantly lower than that of $\mathrm{V}$. Both V and D components are characterised by a symmetric lineshape, as is expected for the case of non-metals such as noble gasses. The small spectral weight of component D (few percent with respect to the saturated layer) and the fact that it remains constant throughout the uptake suggests that it could be originated by Ar atoms adsorbed on Gr defect sites. The V component is instead attributed to Ar adsorbed on the intact epitaxial Gr layer.

The most important step in the characterisation of the $\mathrm{Ar} / \mathrm{Gr} / \operatorname{Ir}(111)$ system was the determination of the adsorption energy of Ar by measuring desorption curves for several initial Ar coverages, i.e. using the same strategy adopted for CO. Figure 2(b) shows a selected Ar 2 $\mathrm{p}_{3 / 2}$ TP-XPS experiment, corresponding to an initial Ar coverage of 0.44 ML. The desorption process starts just below $40 \mathrm{~K}$ and proceeds with a higher rate, until the peak vanishes completely above $43 \mathrm{~K}$. The same analysis which was done to determine the desorption energy of $\mathrm{CO}$ was performed also for all the desorption curves of Ar measured for different initial Ar doses, namely 0.05, 0.15 ML and 0.44 ML, which are shown in Figure 2(c). This analysis yields the same value for both the pre-exponential factor, $\nu=10^{14.8 \pm 0.5} \mathrm{~s}^{-1}$, and for the desorption energy $E_{\mathrm{DES}}=136 \pm 4 \mathrm{meV}$, indicating that the lateral inter-atomic interactions are negligible, unlike the case of CO. This also explains why the fit provides a significantly better description of the experimental data, which is also the reason why the errors associated to the parameters are significantly lower than for the case of CO adsorption. 


\section{Argon Desorption from Graphene/Cobalt/Ir(111)}

Selected core level spectra corresponding to different Ar coverages on Gr/Co/Ir(111) are shown in Figure 2(d). As for the previous experiments the spectra have been acquired during the Ar uptake experiment, which is discussed in the Supporting Information. Also in this case, two components are found at low coverage (Figure 2(d), below), one behaving like the D component described above, at about $242.8 \mathrm{eV}$, the other behaving like the $\mathrm{V}$ component, increasing linearly with the Ar dose. However, in this system, the BE of the $\mathrm{V}$ component is not constant, but decreases by about $100 \mathrm{meV}$ for increasing coverage. At saturation, its core level BE is $243.14 \mathrm{eV}$. A third component $(\mathrm{H})$ appears in the second part of the uptake (Figure 2(d), above), with a BE of about $242.98 \mathrm{eV}$ and shifted from the $\mathrm{V}$ component by $160 \mathrm{meV}$, as was the case for $\mathrm{CO}$ adsorption on the same system.

Finally, desorption curves were measured for different initial coverages of Ar. The desorption curves of the $\mathrm{H}$ and $\mathrm{V}$ components for a saturated Ar layer are shown in Figure 2(f). The $\mathrm{H}$ component desorbs first, with a broad edge starting at about $43 \mathrm{~K}$, and completely desorbs at about $49 \mathrm{~K}$; as for the case of CO desorption from $\mathrm{Gr} / \mathrm{Ir}$, this curve cannot be analysed quantitatively. The desorption of the V component, on the other hand, starts below $50 \mathrm{~K}$ and is complete just above $52 \mathrm{~K}$. The fit yields a pre-exponential factor of $\nu=10^{14.8 \pm 0.5} \mathrm{~s}^{-1}$ and a desorption energy $E_{\mathrm{DES}}=167 \pm 10 \mathrm{meV}$. As for the case of $\mathrm{Ar} / \mathrm{Gr} / \mathrm{Ir}$, the temperature of the desorption process is independent of coverage, and also the low-coverage desorption curves yield a desorption energy of $164 \pm 5 \mathrm{meV}$, comparable to the high-coverage case within the error bar.

\section{Theoretical Results}

The experimental results obtained for $\mathrm{CO}$ and Ar desorption from the two different systems were used as benchmark for our DFT investigations, which were performed using different vdW functionals. For all configurations the best agreement with the experimentally determined values was found in the case of the DF2 approach. 


\section{Structure of Graphene/Ir(111) and Graphene/Co/Ir(111)}

Prior to studying the energetics of the adsorbate systems we investigated the structure of the graphene/Ir(111) and the graphene/Co/Ir(111) interfaces. The relaxation of the $\operatorname{Gr} / \operatorname{Ir}(111)$ structure in the absence of any adsorbate results in a configuration that is characterized by an average separation between the Gr layer and the Ir top-most layer of $3.43 \AA$, close to the interlayer separation in graphite $(3.35 \AA)$, and in good agreement with the reported average height of $3.38 \pm 0.04 \AA$ determined by X-ray standing wave experiments. $\frac{37}{3}$ The Gr layer is observed to exhibit a small corrugation that ranges from a minimum separation of $3.27 \AA$ to a maximum of $3.68 \AA$ from the substrate. The highest separation occurs around a Gr hexagonal ring centered on top of a substrate Ir atom; in what follows we will refer to this region as the "hill" region. In contrast, the minimum separation occurs for a $\mathrm{C}$ atom located directly above an Ir one, in a region denoted as the "valley" region (V). Within the $(10 \times 10)$ Gr supercell there is one hill $(\mathrm{H})$ and one valley $(\mathrm{V})$ region. Our relaxed structure is in good agreement with the experimentally observed moirè pattern characteristic of this system, showing an overall lattice mismatch of about $1 \%$.

We also studied the Gr structure when a layer of Co atoms is added on top of the Ir slab, to simulate the $\mathrm{Gr} / \mathrm{Co} / \operatorname{Ir}(111)$ interface. In agreement with previous findings, $\stackrel{34}{\text { our }}$ calculations show that the the moiré-driven corrugation is largely increased with respect to the $\operatorname{Gr} / \operatorname{Ir}(111)$ case. The $\mathrm{C}$ to substrate distance amounts to $3.15 \AA$ and $1.88 \AA$ in the hills and valley regions, respectively.

\section{Carbon Monoxide on $\mathrm{Gr} / \operatorname{Ir}(111)$ and $\mathrm{Gr} / \mathrm{Co} / \operatorname{Ir}(111)$}

We first consider the adsorption of isolated CO molecules on Gr/Ir(111), as shown in Figure 3(a). Given the corrugation of the Gr layer on the Ir substrate, it is to be expected that there may be small variations of adsorption of $\mathrm{CO}$ in the hill and valley regions. This expectation is indeed proven by our results; in fact, the most stable configuration we have found occurs in the valley region, and is illustrated in Figure 3(b): the CO molecule lies 


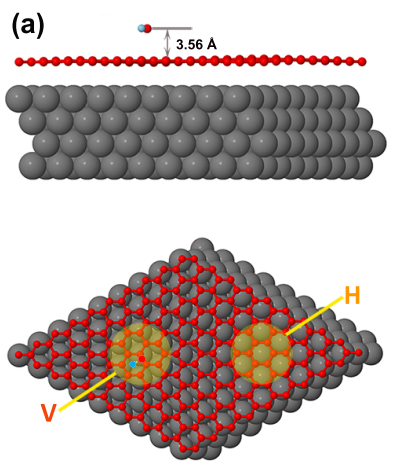

(b)

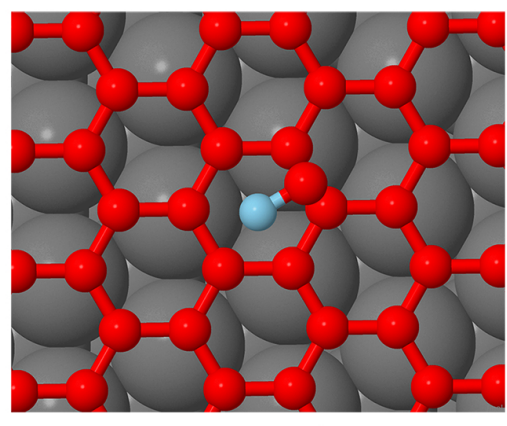

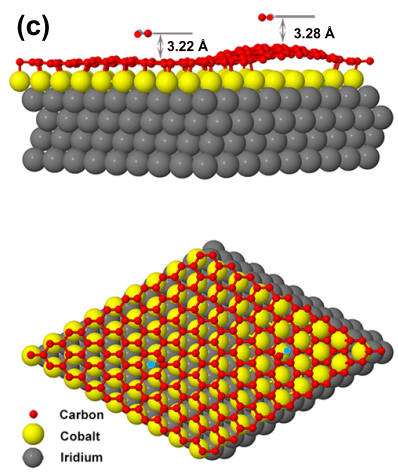

(d)

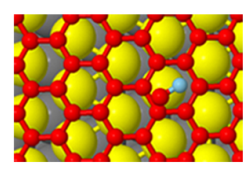

(e)

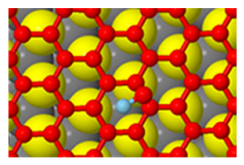

Figure 3: (a) Side and top views of the of the most stable CO adsorption configuration as found in our calculations. Grey atoms represent the $\operatorname{Ir}(111)$ surface; carbon atoms are displayed in red, and the oxygen atom is shown in blue. Valley and Hill regions are coloured in the top view. (b) Local configuration of the $\mathrm{CO}$ molecule, with the $\mathrm{O}$ atom sitting above the centre of a $\mathrm{Gr}$ hexagon and the $\mathrm{C}$ atom located in a bridge site. (c) Top and side views of the minimum energy CO adsorption configurations on the hill and valley of the Gr moirè unit cell on $\mathrm{Co} / \operatorname{Ir}(111)$. Cobalt atoms are shown in yellow. (d-e) Local configuration of the adsorption configuration of CO in both valley (d) and hill (e) regions on $\mathrm{Gr} / \mathrm{Co} / \mathrm{Ir}(111)$.

approximately flat on the surface, with the oxygen atom located above the centre of a $\mathrm{Gr}$ hexagon, while the carbon atom is located over a bridge site of the Gr honeycomb. The center of the $\mathrm{CO}$ molecule is at a distance of $3.56 \AA$ above the Gr layer with the molecular axis lying almost flat on the surface.

The adsorption energy calculated by using the DF2 approach is $146 \pm 2 \mathrm{meV}$, which is in much better agreement with the experimental result $(146 \mathrm{meV})$ than the value calculated using the D3 functional (194 meV). In total, we have have considered and relaxed more than twenty initial configurations of the adsorbate, distributed over the $(10 \times 10)$ Gr supercell. We find that binding tends to be slightly stronger in the valley region, as happens in the case of atomic clusters, ${ }^{38}$ with an average binding energy which is about 5 meV larger than in the hill region; even if small, this difference is systematic. We also studied initial configurations in which the adsorbate was placed with its axis perpendicular to the surface, with either the oxygen or carbon atom pointing towards the surface. These initial configurations also resulted in stable relaxed structures retaining the verticality of the adsorbate, but these were less strongly bound to the substrate, typically by $40 \mathrm{meV}$. In all the absorbed configurations, the CO bond distance is $1.145 \AA$, essentially unchanged from the gas-phase value, obtained 
by performing a calculation for the isolated CO molecule in the same simulation box, without the substrate.

An interesting issue to consider is the role of dispersion-type interactions. To address this point, we simply performed relaxation calculations of the adsorbate on the substrate, as before, but without including the dispersion interactions. The Gr and metal substrate atoms were constrained to remain at their previously relaxed positions, and only the adsorbate was allowed to relax. As expected, without van der Waals interactions the binding of the CO adsorbate to the surface becomes negligible (approx. $20 \mathrm{meV}$ ). The distance of the molecule to the surface increases by $0.3-0.4 \AA$; the oxygen atom moves further away.

Up to this point, we have considered the adsorption of single CO molecules, but the polar nature of the adsorbate leads one to expect that the binding energy may have a noticeable dependence on coverage, as is the case for example for CO adsorption on graphite(0001). To investigate this issue, we have performed relaxation calculations on adsorbed clusters of $\mathrm{CO}$ molecules of various sizes, which confirmed that the adsorption energy slightly increases with coverage, as reported in the Supporting Information.

Compared to the adsorption on $\mathrm{Gr} / \operatorname{Ir}(111)$, the preferred adsorption site of $\mathrm{CO}$ on $\mathrm{Gr} / \mathrm{Co} / \operatorname{Ir}(111)$ is almost unaltered, in a valley region (see Figure $3(\mathrm{c})$ ). While the molecule moves slightly towards the centre of the Gr hexagon, its centre of mass remains inside a Gr hexagon, with the CO molecular axis parallel to the surface. The adsorption energy is $168 \mathrm{meV}$, a value higher by about $30 \mathrm{meV}$ with respect to that on $\mathrm{Gr} / \operatorname{Ir}(111)$. On the other hand, the adsorption energy in the hill region is $123 \mathrm{meV}$. This difference of about $45 \mathrm{meV}$ is clearly larger than the one between the hills and valleys of $\operatorname{Gr} / \operatorname{Ir}(111)$, which was only about $5 \mathrm{meV}$, which suggest a possible combined contribution due to the closer distance to the substate and the effect of the graphene corrugation. In this respect it is interesting to note that the CO to graphene distance for the isolated molecules best adsorption configurations in the hills and in the valleys is almost the same, being $3.22 \AA$ and $3.28 \AA$, respectively. As for $\mathrm{CO}$, also for the Ar adsorption energy the agreement with the experimental values in 
the valley $(180 \mathrm{meV})$ is in better agreement with respect to the value determined by using the D3 functional (203 meV). Based on our benchmarks, all the values reported in the next paragraph have been calculated using the DF2 functional.

\section{Argon on $\mathrm{Gr} / \operatorname{Ir}(111)$ and $\mathrm{Gr} / \mathrm{Co} / \operatorname{Ir}(111)$}

The corrugated Gr layer plays an important role also for values of the Ar adsorption energy. Calculated adsorption energies of Ar atoms in the valleys of $\mathrm{Gr} / \mathrm{Co} / \mathrm{Ir}(111)(159 \mathrm{meV})$ are 40 meV larger than in the case of the hills $(120 \mathrm{meV})$, showing a difference which is comparable to the one found for CO in the same system. The agreement between experiment and theory is very good also in the case of Ar adsorption in the valleys of $\mathrm{Gr} / \mathrm{Co} / \mathrm{Ir}(111)$, the difference being only $5 \mathrm{meV}$.

As for the case of $\mathrm{CO}$ adsorption, for $\mathrm{Ar}$ on $\mathrm{Gr} / \mathrm{Ir}(111)$, the very large adsorbate-metal $(7.46 \AA)$ and adsorbate-graphene $(4.02 \AA)$ distance is reflected in a reduced adsorption energy $(106 \mathrm{meV})$, which differs from the experimental value by about $30 \mathrm{meV}$.

\section{Interaction of $\mathrm{CO}$ and Ar with Gr or the Metal Substrate Only}

Finally, we have investigated the causes and mechanisms of this substrate-induced difference in adsorption energy. In fact, as already reported, this effect could be explained either by a direct interaction of $\mathrm{CO}$ with the metal below graphene (direct interaction), which would imply that graphene is translucent or transparent to vdW forces, $\stackrel{1112}{12}$ or to an indirect mechanism. $\frac{14}{14}$ This, in turn, could be related either to the altered geometry and bond length of graphene when it is corrugated, or to the charge transfer and redistribution induced by the interaction with the substrate, $\frac{8[34 \mid 41}{}$ which is known to induce doping and changes to the work function and potential energy surface not only of Gr, but also of its substrate. $\underline{42} \underline{44}$

To decouple these different contributions and identify the mechanisms by which the substrate influences the adsorption properties of graphene, we have performed further theoretical calculations, in addition to the ones performed on the systems experimentally investigated 


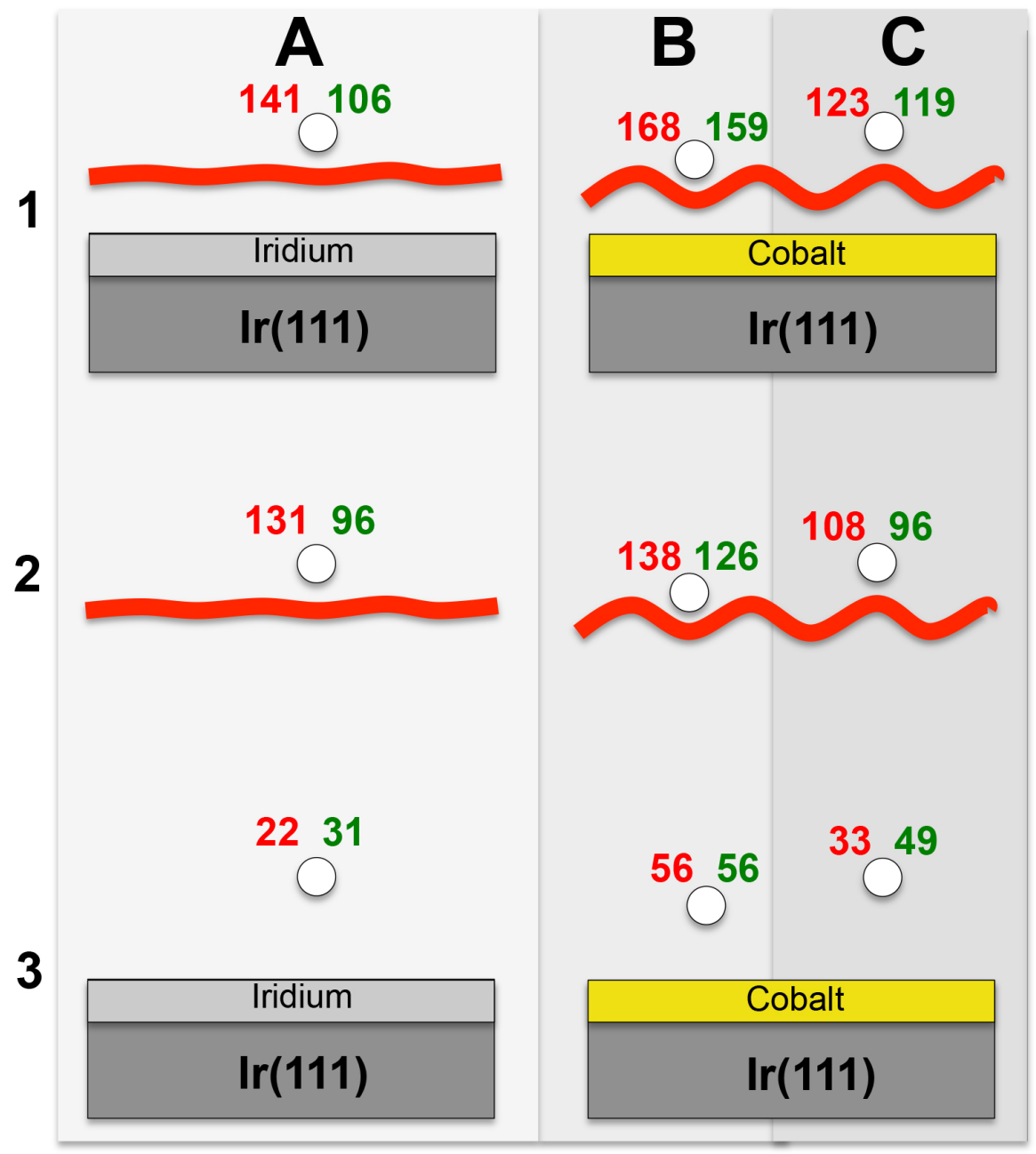

Figure 4: Interaction of $\mathrm{CO}$ (red value, on the left) and $\mathrm{Ar}$ (green value, on the right) adsorbates for three different adsorption configurations (A: on flat graphene; B and C: in the valleys and hills (respectively) of corrugated graphene) with the Gr/metal system (1) and with the graphene (2) and metal (3) alone.

(i.e. 1A, B and C), as shown in Figure 4 and in the Supporting Information. These calculations were performed by keeping all distances (adsorbate-Gr and adsorbate-substrate) fixed to the value found for the corresponding real systems, i.e. the distance of flat Gr was fixed to the one it has from $\operatorname{Ir}(111)$ and that of corrugated Gr to the one it has from $\mathrm{Co} / \operatorname{Ir}(111)$.

In particular, to investigate the role of graphene in screening the direct interactions between the adsorbates and the substrate, we separately calculated the adsorption energy for each adsorbate placed either on a graphene sheet with the substrate removed, or on the substrate alone, without graphene, for both the flat Gr on Ir and corrugated Gr layer on Co. 
The results are shown in Figure 4 for the case of both $\mathrm{CO}$ molecules (red values) and Ar atoms (green values).

The results show that the adsorption energy between the adsorbates and the isolated Gr layer (2) vary between about 100 to $140 \mathrm{eV}$ in all systems, with the lowest values found for the hills and the highest ones for the valleys of the corrugated layer, while the flat layer shows an intermediate behaviour. The direct interaction of the adsorbates with the substrate is significantly lower, between about 20 and $55 \mathrm{eV}$, which is due to the much larger separation between the atoms or molecules and the substrate, in the geometry analysed.

\section{Discussion}

The adsorption energy of both $\mathrm{CO}$ and $\mathrm{Ar} / \mathrm{Gr}$, calculated with the vdW-DF2 functional, shows a very good agreement with the experimental results. The theoretically calculated models show that the preferred adsorption site for a single CO molecule and Ar atom is in the valley of the $\mathrm{Gr} / \mathrm{Co} / \operatorname{Ir}(111)$ supercell, while the hills are only populated at high coverage. In this respect, the presence of two $\mathrm{C}$ 1s and $\mathrm{Ar} 2 \mathrm{p}_{3 / 2}$ photoemission components which have been observed for both, $\mathrm{CO}$ and $\mathrm{Ar}$ adsorbed on $\mathrm{Gr} / \mathrm{Co} / \mathrm{Ir}$ respectively(Figure 2(a-c)) are attributed to molecules adsorbed in the valleys ( $\mathrm{V}$ component) and hills ( $\mathrm{H}$ component) of the corrugation.

It is interesting to compare these results to the adsorption of $\mathrm{Ar}$ on the $\mathrm{Gr} / \mathrm{Ru}(0001)$ surface. ${ }^{45}$ In that case, the BE reported for the $\mathrm{V}$ and $\mathrm{H}$ components is 243.16 and $242.86 \mathrm{eV}$ respectively. These values and the shift between them are very close to the ones obtained in our experiment, showing that the effects of the interaction of $\mathrm{Ru}$ and $\mathrm{Co}$ with $\mathrm{Gr}$ is quite similar. Furthermore, the higher $\mathrm{C}$ 1s BE of the $\mathrm{V}$ component with respect to the $\mathrm{H}$ component reflects the higher $\mathrm{C}$ 1s $\mathrm{BE}$ of the graphene atoms closest to the surface in $\mathrm{Gr} / \mathrm{Co} / \mathrm{Ir}(111) . \underline{34}$ This similarly explains the overall higher BEs of both the C 1s from CO and $\operatorname{Ar} 2 \mathrm{p}_{3 / 2}$, in the former system, where the $\mathrm{C} 1 \mathrm{~s}$ BE of graphene is higher by more than $500 \mathrm{meV}$ than in $\mathrm{Gr} / \mathrm{Ir}(111)$. It is also interesting to note that if we take as a threshold the 
value of $2.2 \AA$ (maximum height of the $\mathrm{C}$ atoms in the flat region (valley) of the Gr layer on $\mathrm{Co} / \operatorname{Ir}(111)$ interface), $61 \%$ of the Gr unit cell is in the flat region. This nicely compares with the populations of the $\mathrm{CO}$ molecules or $\mathrm{Ar}$ atoms in the valleys as found by quantitative analysis for the $\mathrm{CO}$ saturated layer on $\mathrm{Gr} / \mathrm{Co} / \mathrm{Ir}(111)$ (see mentioned in the experimental results). Following these considerations, the broad desorption edge of the $\mathrm{H}$ component is due to the wide distribution of adsorption sites on the hill regions where the distance of the C atoms of Gr from the substrate varies strongly due to the enhanced corrugation of Gr after intercalation. This leads to a broad distribution of the adsorption energy of molecules and atoms in these non-equivalent sites observed in the desorption curve of the $\mathrm{H}$ component. This behaviour has been observed also for Xe adsorption on corrugated Gr. ${ }^{46}$

Although the Gr layer displays valley and hill regions when deposited on both the $\operatorname{Ir}(111)$ and $\mathrm{Co} / \operatorname{Ir}(111)$ substrates, it is obvious from Figure 5 that the level of Gr corrugation is significantly larger in the latter case. This is reflected by the fact that only one $\mathrm{C}$ is component from $\mathrm{CO}$ and $\mathrm{Ar} 2 \mathrm{p}_{3 / 2}$ from Ar adsorbed on $\mathrm{Gr} / \operatorname{Ir}(111)$ is observed (Figures 1 and 2), even at high coverage, while two are present in the case of $\mathrm{CO}$ on $\mathrm{Gr} / \mathrm{Co} / \operatorname{Ir}(111)$.

It is evident from our results that a significant difference in the adsorption energy of both $\mathrm{CO}$ and $\mathrm{Ar}$ is observed between a flat and a corrugated, free-standing Gr layer, i.e. in absence of the metal substrate (see Figure 4). However, this does not account alone for the experimental observations as the calculated adsorption energy on the isolated layer is significantly lower than the one measured on the metal-supported Gr. This indicates that there is indeed an effect of the metal substrate on the adsorption properties of Gr.

To verify whether the model of transparency or translucency originally proposed for water adsorption can also hold for the adsorption of $\mathrm{CO}$ and $\mathrm{Ar}$, we expressed the total interaction $I$ of the adsorbates with the Gr/metal system as

$$
I=G+k S
$$




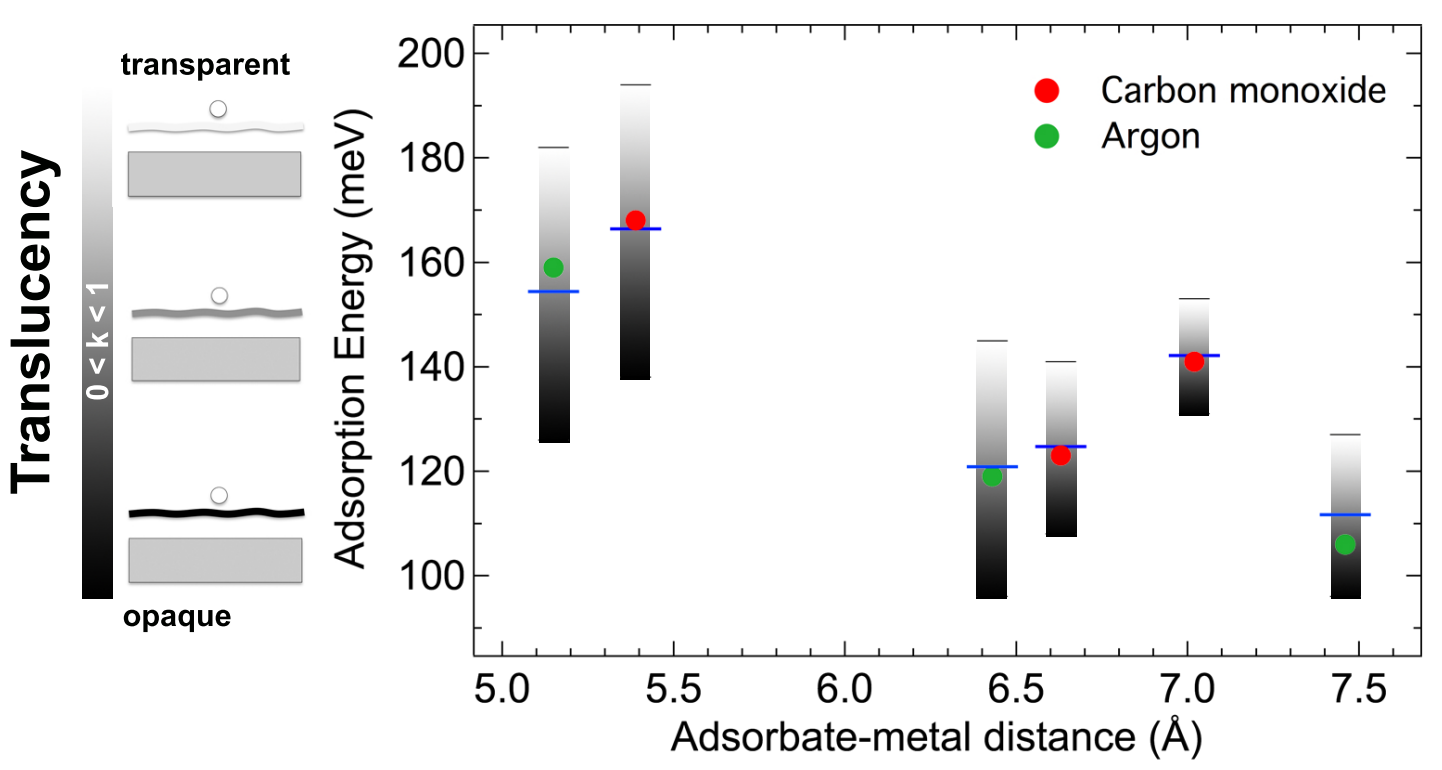

Figure 5: Adsorption energy dependence on adsorbate-metal distance for carbon monoxide molecules (red) and argon atoms (green) in the different configurations discussed in Figure 4, i.e. on flat Gr and on the hills and valleys of corrugated Gr. Blue ticks indicate the results of best fit using the equation $\mathrm{I}=\mathrm{G}+\mathrm{kS}$, where $k$ is the factor accounting for the screening of the $\mathrm{vdW}$ interactions by Gr. Vertical bars correspond to the values of complete blocking (black region) and total transparency (white region) of Gr.

where $G$ is the interaction with the free-standing Gr layer, $S$ is the interaction with the metal substrate in absence of Gr, but at the same distance (see Figure 4), and $0 \leq k \leq 1$ accounts for the screening of the $\mathrm{vdW}$ interactions by Gr. The case $k=0$ would correspond to a complete blocking of the vdW interactions by Gr, while $k=1$ to a total transparency of Gr to vdW interactions.

In Figure 5, we show the contributions of $\mathrm{Gr}$ and of the substrate as a function of the translucency parameter $k$, for both $\mathrm{CO}$ and $\mathrm{Ar}$ on flat $\mathrm{Gr}$ and on the hills and valleys of corrugated Gr. The adsorption energy of all the systems is described with high accuracy with translucency $k$ of $0.507 \pm 0.034$. This number can be compared with the value of about 0.30 which was found in the case of water-substrate interaction using the contact angle approach. ${ }^{122}$ It is important to underline that our approach to measure the adsorption energies is based on a direct method which overcomes all the problems inherent to the contact angle approach, which is typically used to probe the adsorption energy of water on Gr. 
Finally, to further prove that the translucency we observe is indeed due to vdW forces (and therefore a "real" translucency), we exchanged the first layer metal composition (Co instead of Ir and Ir instead of Co), while the Gr, the metal substrate atoms and Carbon Monoxide were constrained to remain at their previously relaxed positions (see Supporting Information). In this way, we verified that the effect of the different degree of charge transfer between Gr and its substrate, which is known to change because of the different chemical identity of the supporting substrate, is negligible when compared to the direct dispersive contribution from the substrate, which is related to the adsorbate-graphene and adsorbatemetal distances as it is based on the $\mathrm{vdW}$ interactions. In fact, the results show that the adsorption energy is approximately the same, and does not change because of the modification of the chemical composition of the substrate, while it has a strong dependence on the distance: this is a further proof that the role of the substrate on the adsorption energy is due to a direct vdW coupling.

The translucency of Gr to vdW interactions, obtained in our investigation, follows from the fact that dispersion forces are known to be non-additive. Transparency would imply a value $k=1$ in the above equation, while our results clearly show that the actual value obtained is closer to 0.5 . Our results show that this value applies to two different metal substrates and two different adsorbates, including noble gas atoms and a molecule having polar nature.

\section{Conclusions}

We described a combined experimental and theoretical investigation of the adsorption energy of carbon monoxide and argon on high-quality epitaxial graphene supported by different metal substrates. By comparing two systems characterized by a different chemical composition of the substrate, we have proved that the adsorption energy of $\mathrm{CO}$ and Ar on graphene is significantly increased (by about 20\%) when graphene is strongly interacting with the 
substrate, with respect to a weakly interacting case. We have investigated the mechanisms leading to the enhancement in the molecular and atomic adsorption energy by decoupling the different effects of the substrate and of graphene. In addition, we have shown that the corrugation of graphene plays an important role being the convex adsorption region of graphene the one where the desorption energy is larger.

Our results show that the molecule/atom adsorption energy is dominated by the interaction with the graphene layer, which is closer to the adsorbates, but it is also influenced directly by the long range interaction with the metal substrate. In this respect graphene acts as a translucent medium which is capable to produce a $50 \%$ screening of the van der Waals forces. Our findings provide fundamental informations on the interaction of graphene with adsorbates and on the effect of the substrate supporting graphene, and can be used to optimize its properties for applications, especially in the field of sensoristics and metal-free catalysis.

\section{Methods}

\section{Experimental}

We have characterized different coverages of $\mathrm{CO}$ and Ar adsorbed on Gr by high energyresolution X-ray photoelectron spectroscopy (XPS), near edge X-ray absorption fine structure spectroscopy (NEXAFS) and low energy electron diffraction (LEED). All experiments were performed at the SuperESCA beamline ${ }^{47}$ of the Elettra synchrotron radiation facility in Trieste, Italy, in an experimental chamber with a residual background pressure always in the low $10^{-10}$ mbar range. The $\operatorname{Ir}(111)$ single crystal was mounted on a manipulator with 4 degrees of freedom. The sample was heated either by direct irradiation or by electron bombardment from three tungsten filaments mounted behind it. The sample was cooled using a liquid-helium filled cryostat: the temperature was measured using two K-type thermocouples directly spot-welded to the crystal. The temperature was calibrated using two methods: 
by fitting the shape of the Fermi edge photoemission spectrum to the Fermi-Dirac function - convoluted with a Gaussian taking into account the experimental contribution - and by measuring the temperature of the desorption onset of Ar gas multilayers, which is known to start at $25 \mathrm{~K} \cdot \frac{48}{4}$ The overall temperature calibration is affected by an error of $\pm 1 \mathrm{~K}$.

The $\mathrm{Gr} / \operatorname{Ir}(111)$ and $\mathrm{Gr} / \mathrm{Co} / \operatorname{Ir}(111)$ interfaces were prepared using well established procedures, reported in the Supporting Information. The quality of graphene was tested by means of high-resolution low energy electron diffraction Spot Profile Analysis-LEED which show the typical moirè pattern and a full width at half maximum of the diffraction spots which indicates that graphene flakes are as large on average as $300 \AA$ x $300 \AA$, which would indicate that domain boundaries account for at most $1 \%$ of the total carbon atoms. In addition, we can safely rule out the formation of a high density of graphene defects such as single-vacancies or Stone-Wales defects. Based on DFT calculations performed on both Highly Oriented Pyrolitic Graphite ${ }^{\sqrt{49}}$ and graphene,, 50 the presence of this kind of defects in a concentration higher than $1 \%$ would clearly show up in our high-resolution core level spectra in the form of lower binding energy components.

Each gas was dosed onto the sample from a leak valve at a constant pressure of $5 \times 10^{-9} \mathrm{mbar}$, as obtained after correction for the base pressure and gauge-specific sensitivity factor. The coverage was measured in Monolayers $(1 \mathrm{ML}=$ number of adsorbed $\mathrm{CO}$ molecules or Ar atoms per graphene unit cell): based on this definition, for example, a saturated layer of $\mathrm{CO}$, which forms a $(\sqrt{3} \times \sqrt{3}) \mathrm{R} 30^{\circ}$ structure with one molecule every three Gr unit cells, corresponds to a $1 / 3 \mathrm{ML}$ coverage, i.e. $6.36 \times 10^{14} \mathrm{molecules} / \mathrm{cm}^{2}$. The coverage was estimated based on the ideal coverage associated to the ordered structures formed by each adsorbate, either CO or Ar, on graphene, as probed by LEED (see Supporting Information). Even if this method cannot provide an accurate estimate, this is not relevant for this study as the desorption dynamics for both species were found to follow a first-order process, which is independent of coverage. In addition, for the case of $\mathrm{CO}$, the coverage was verified by comparing the area of the background-subtracted C 1s photoemission peaks 
of clean graphene (whose $\mathrm{C}$ atoms density corresponds to that of $2 \mathrm{ML} \mathrm{CO}$ ) and of each $\mathrm{CO}$ exposure, based on the fact that as long as the coverage is lower than a full layer, the photoemission signal is to a first approximation linearly proportional to coverage. The labels $\mathrm{V}$ and $\mathrm{H}$ will be used to indicate the atomic/molecular adsorption in the valleys and in the hills of the corrugated graphene unit cell.

Fast X-ray photoemission spectra were measured either in snap-shot mode $\sqrt{51}$ or in scanning-mode during the uptake (up to different coverages) and desorption experiments, with a data acquisition time down to $500 \mathrm{~ms} /$ spectrum, depending on the specific core level. High energy-resolution spectra were taken in scanning mode, with a longer dwell time and smaller electron binding energy (BE) steps, after each gas exposure. The $\mathrm{C}$ 1s and Ar 2 $\mathrm{p}_{3 / 2}$ spectra were measured using a photon energy of $325 \mathrm{eV}$ and $400 \mathrm{eV}$ respectively, in normal emission conditions, with an overall experimental resolution of $50 \mathrm{meV}$. The BE scale was calibrated with respect to the Fermi level. The exposure in the experiments was measured in Langmuir, being $1 \mathrm{~L}=10^{-6}$ torr $\times 1 \mathrm{~s}$.

Possible photon-beam induced desorption effects were carefully investigated by moving the Ir crystal by a distance larger than the photon beam size at the sample (vertical dimension $5 \mathrm{\mu m}$ ) after dosing a full layer of both CO and Ar: even though such an effect could be detected, especially for the case of CO $(0.5 \%$ of ML undergoes photon-induced desorption after $1000 \mathrm{~s}$ X-ray exposure at $\mathrm{h} \nu=325 \mathrm{eV}$ ), it was only relevant on a time scale which was much larger than our data acquisition time per spectrum. An even slower process was observed for Ar. Therefore, all photoemission spectra of both CO and Ar were measured while moving the crystal in steps of $30 \mu \mathrm{m}$ (i.e. about 6 times the size of the beam) perpendicularly to the beam every $30 \mathrm{~s}$.

All photoemission spectra were fitted to a sum of Donjach-Šunjić (DS) lineshapes $\frac{52}{-}$ characterized by a Lorentzian full width at half maximum (FWHM) $L$, which takes into account the effect of finite core-hole lifetime, and by the asymmetry index $\alpha$, which describes the low-energy electron hole pair excitations near the Fermi level — convoluted with 


\section{Theoretical}

In order to shed light on the experimental results, we have performed theoretical calculations on a series of structural models of $\mathrm{CO}$ or Ar adsorbed on $\mathrm{Gr} / \operatorname{Ir}(111)$ and $\mathrm{Gr} / \mathrm{Co} / \operatorname{Ir}(111)$. We have used density functional theory $\sqrt{55156}$ to model the system as implemented in the VASP code. ${ }^{57}$ We employed the projector-augmented wave (PAW) method ${ }^{58159}$ to account for the core electrons; we included 9 electrons $\left(5 \mathrm{~d}^{7}, 6 \mathrm{~s}^{2}\right)$ per Ir, 9 electrons $\left(3 \mathrm{~d}^{7}, 4 \mathrm{~s}^{2}\right)$ per Co, 4 per $\mathrm{C}$ and 6 per $\mathrm{O}$ atom explicitly in the valence. Exchange-correlation effects were incorporated with the revPBE generalized-gradient functional. ${ }^{60161}$ Because in the adsorption of $\mathrm{CO}$ and Ar on the $\mathrm{Gr} / \operatorname{Ir}(111)$ and $\mathrm{Gr} / \mathrm{Co} / \operatorname{Ir}(111)$ substrates we expect dispersion forces to play a key role, we used three different functionals, as implemented in the framework of D3, 62 Langreth $h^{\sqrt{63}[65}$ and DF2 ${ }^{66}$ formalisms.

In fact, the measured adsorption energies have been used as benchmark to understand which vdW functional was the most accurate to describe the experimental findings. KohnSham orbitals were represented by means of plane-waves up to a kinetic energy cutoff of 400 
eV. Brillouin zone sampling was limited to the Gamma point.

We used a slab geometry consisting of four layers of $(9 \times 9) \operatorname{Ir}(111)$ cells, with the bottom two layers held fixed at the equilibrium bulk positions. On the free surface side of the slab we placed a $(10 \times 10)$ supercell graphene layer, which results in a supercell size very close to its experimental value. $\stackrel{37}{ }$ In total, the resulting slab contains 524 atoms $(324 \mathrm{Ir}+200 \mathrm{C})$. The size of the cell in the direction perpendicular to the substrate plane was set to $24.4 \AA$. For the $\mathrm{Gr} / \mathrm{Co} / \operatorname{Ir}(111)$ system, additional $(9 \times 9)$ cells of Co atoms were placed in between the Ir and Gr. On the resulting slab, we placed a single CO molecule or Ar atom. All structures were fully relaxed using the conjugate gradients method, until forces on all atoms were smaller than $0.01 \mathrm{eV} / \AA$.

\section{Supporting Information Available}

Sample cleaning, Gr growth and Co intercalation experimental procedures. Vibrationally resolved photoemission spectra of $\mathrm{CO} / \mathrm{Gr}$. NEXAFS dichroism to probe the orientation of adsorbed molecules. LEED pattern at saturation coverage. Details on the data analysis and error estimation procedure used for the desorption curves. Additional DFT calculations. This material is available free of charge on the ACS Publications website at DOI: xxxx.

\section{Acknowledgement}

ERH and AG thank MICINN for funding this research through project FIS2015-64222-C21-P. Part of the calculations were performed on the UK National service ARCHER, and part in the Finis Terrae II machine at CESGA. We thank L. Bignardi and D. Curcio for their contribution to the preliminary measurements. 


\section{References}

1. Primo, A.; Parvulescu, V.; Garcia, H. Graphenes as Metal-Free Catalysts with Engineered Active Sites. J. Phys. Chem. Lett. 2017, 8, 264-278.

2. Liang, Y.; Wang, H.; Casalongue, H. S.; Chen, Z.; Dai, H. $\mathrm{TiO}_{2}$ Nanocrystals Grown on Graphene As Advanced Photocatalytic Hybrid Materials. Nano Res. 2010, 3, 701-705.

3. Khalid, N.; Ahmed, E.; Hong, Z.; Sana., L.; Ahmed, M. Enhanced Photocatalytic Activity of Graphene- $\mathrm{TiO}_{2}$ Composite under Visible Light Irradiation. Curr. Appl. Phys. 2013, 13, 659-663.

4. Li, X.; Yu, J.; Wageh, S.; Al-Ghamdi, A. A.; Xie, J. Graphene in Photocatalysis: A Review. Small 2016, 12, 6640-6696.

5. Fasciani, C.; Lanterna, A. E.; Giorgi, J. B.; Scaiano, J. C. Visible Light Production of Hydrogen by Ablated Graphene: Water Splitting or Carbon Gasification? J. Am. Chem. Soc. 2017, 139, 11024-11027.

6. Schedin, F.; Geim, A. K.; Morozov, S. V.; Hill, E. W.; Blake, P.; Katsnelson, M. I.; Novoselov, K. S. Detection of Individual Gas Molecules Adsorbed on Graphene. Nat. Mater. 2007, 6, 652-655.

7. Novoselov, K.; Fal'Ko, V.; Colombo, L.; Gellert, P.; Schwab, M.; Kim, K. A Roadmap for Graphene. Nature 2012, 490, 192-200.

8. Batzill, M. The Surface Science of Graphene: Metal Interfaces, CVD Synthesis, Nanoribbons, Chemical Modifications, and Defects. Surf. Sci. Rep. 2012, 67, 83-115.

9. Not so Transparent. Nat. Mater. 2013, 12, 865, Editorial.

10. Belyaeva, L. A.; van Deursen, P. M.; Barbetsea, K. I.; Schneider, G. F. Hydrophilicity of Graphene in Water through Transparency to Polar and Dispersive Interactions. Adv. Mater. 2018, 30, 1703274. 
11. Rafiee, J.; Mi, X.; Gullapalli, H.; Thomas, A. V.; Yavari, F.; Shi, Y.; Ajayan, P. M.; Koratkar, N. A. Wetting Transparency of Graphene. Nat. Mater. 2012, 11, 217-222.

12. Shih, C.-J.; Strano, M. S.; Blankschtein, D. Wetting Translucency of Graphene. Nat. Mater. 2013, 12, 866-869.

13. Raj, R.; Maroo, S. C.; Wang, E. N. Wettability of Graphene. Nano Lett. 2013, 13, 1509-1515.

14. Chakradhar, A.; Sivapragasam, N.; Nayakasinghe, M. T.; Burghaus, U. Support Effects in the Adsorption of Water on CVD Graphene: An Ultra-High Vacuum Adsorption Study. Chem. Commun. (Cambridge, U. K.) 2015, 51, 11463-11466.

15. Hong, G.; Han, Y.; Schutzius, T.; Wang, Y.; Pan, Y.; Hu, M.; Jie, J.; Sharma, C.; Müller, U.; Poulikakos, D. On the Mechanism of Hydrophilicity of Graphene. Nano Lett. 2016, 16, 4447-4453.

16. An, S.; Joshi, B. N.; Lee, J.-G.; Lee, M. W.; Kim, Y. I.; Kim, M.-W.; Jo, H. S.; Yoon, S. S. A Comprehensive Review on Wettability, Desalination, and Purification Using Graphene-Based Materials at Water Interfaces. Catal. Today 2017, 295, 14-25.

17. Driskill, J.; Vanzo, D.; Bratko, D.; Luzar, A. Wetting Transparency of Graphene in Water. J. Chem. Phys. 2014, 141, 18C517.

18. Amadei, C. A.; Lai, C.-Y.; Esplandiu, M. J.; Alzina, F.; Vecitis, C. D.; Verdaguer, A.; Chiesa, M. Elucidation of the Wettability of Graphene through a Multi-Length-Scale Investigation Approach. $R S C$ Adv. 2015, 5, 39532-39538.

19. Ashraf, A.; Wu, Y.; Wang, M. C.; Yong, K.; Sun, T.; Jing, Y.; Haasch, R.; Aluru, N.; Nam, S. Doping-Induced Tunable Wettability and Adhesion of Graphene. Nano Lett. 2016, 16, 4708-4712. 
20. Du, F.; Huang, J.; Duan, H.; Xiong, C.; Wang, J. Wetting Transparency of Supported Graphene Is Regulated by Polarities of Liquids and Substrates. Appl. Surf. Sci. 2018, $454,249-255$.

21. Hill, C. M.; Kim, J.; Bodappa, N.; Bard, A. J. Electrochemical Nonadiabatic Electron Transfer via Tunneling to Solution Species through Thin Insulating Films. J. Am. Chem. Soc. 2017, 139, 6114-6119.

22. Smerieri, M.; Celasco, E.; Carraro, G.; Lusuan, A.; Pal, J.; Bracco, G.; Rocca, M.; Savio, L.; Vattuone, L. Enhanced Chemical Reactivity of Pristine Graphene Interacting Strongly with a Substrate: Chemisorbed Carbon Monoxide on Graphene/Nickel(1 111$)$. ChemCatChem 2015, 7, 2328-2331.

23. Ambrosetti, A.; Silvestrelli, P. L. Communication: Enhanced Chemical Reactivity of Graphene on a Ni(111) Substrate. J. Chem. Phys. 2016, 144, 111101.

24. Zhu, L.; Zhang, W.; Zhu, J.; Cheng, D. Ni (111)-Supported Graphene As a Potential Catalyst for High-Efficient CO Oxidation. Carbon 2017, 116, 201-209.

25. Kong, W.; Li, H.; Qiao, K.; Kim, Y.; Lee, K.; Nie, Y.; Lee, D.; Osadchy, T.; Molnar, R.; Gaskill, D.; Myers-Ward, R.; Daniels, K.; Zhang, Y.; Sundram, S.; Yu, Y.; Bae, S.; Rajan, S.; Shao-Horn, Y.; Cho, K.; Ougazzaden, A. et al. Polarity Governs Atomic Interaction through Two-Dimensional Materials. Nat. Mater. 2018, 17, 999-1004.

26. Lacovig, P.; Pozzo, M.; Alfè, D.; Vilmercati, P.; Baraldi, A.; Lizzit, S. Growth of DomeShaped Carbon Nanoislands on $\operatorname{Ir}(111)$ : The Intermediate between Carbidic Clusters and Quasi-Free-Standing Graphene. Phys. Rev. Lett. 2009, 103, 166101.

27. Celasco, E.; Carraro, G.; Smerieri, M.; Savio, L.; Rocca, M.; Vattuone, L. Influence of Growing Conditions on the Reactivity of Ni Supported Graphene Towards CO. J. Chem. Phys. 2017, 146, 104704. 
28. Balog, R.; Jørgensen, B.; Wells, J.; Lægsgaard, E.; Hofmann, P.; Besenbacher, F.; Hornekær, L. Atomic Hydrogen Adsorbate Structures on Graphene. J. Am. Chem. Soc. 2009, 131, 8744-8745.

29. Böttcher, S.; Vita, H.; Weser, M.; Bisti, F.; Dedkov, Y. S.; Horn, K. Adsorption of Water and Ammonia on Graphene: Evidence for Chemisorption from X-Ray Absorption Spectra. J. Phys. Chem. Lett. 2017, 8, 3668-3672.

30. Pletikosić, I.; Kralj, M.; Pervan, P.; Brako, R.; Coraux, J.; N’Diaye, A.; Busse, C.; Michely, T. Dirac Cones and Minigaps for Graphene on $\operatorname{Ir}(111)$. Phys. Rev. Lett. 2009, 102, 056808-056811.

31. Balog, R.; Jørgensen, B.; Nilsson, L.; Andersen, M.; Rienks, E.; Bianchi, M.; Fanetti, M.; Lægsgaard, E.; Baraldi, A.; Lizzit, S.; Sljivancanin, Z.; Besenbacher, F.; Hammer, B.; Pedersen, T. G.; Hofmann, P.; Hornekær, L. Bandgap Opening in Graphene Induced by Patterned Hydrogen Adsorption. Nat. Mater. 2010, 9, 315-319.

32. Baraldi, A.; Comelli, G.; Lizzit, S.; Cocco, D.; Paolucci, G.; Rosei, R. Temperature Programmed X-Ray Photoelectron Spectroscopy: A New Technique for the Study of Surface Kinetics. Surf. Sci. 1996, 367, L67-L72.

33. Pacilé, D.; Lisi, S.; Di Bernardo, I.; Papagno, M.; Ferrari, L.; Pisarra, M.; Caputo, M.; Mahatha, S.; Sheverdyaeva, P.; Moras, P.; Lacovig, P.; Lizzit, S.; Baraldi, A.; Betti, M.; Carbone, C. Electronic Structure of Graphene/Co Interfaces. Phys. Rev. B 2014, 90, $195446-195451$.

34. Presel, F.; Jabeen, N.; Pozzo, M.; Curcio, D.; Omiciuolo, L.; Lacovig, P.; Lizzit, S.; Alfè, D.; Baraldi, A. Unravelling the Roles of Surface Chemical Composition and Geometry for the Graphene-Metal Interaction through C1s Core-Level Spectroscopy. Carbon 2015, 93, 187-198. 
35. Wang, Z.; Seebauer, E. Estimating Pre-Exponential Factors for Desorption from Semiconductors: Consequences for A Priori Process Modeling. Appl. Surf. Sci. 2001, 181, $111-120$.

36. Campbell, C. T.; Árnadóttir, L.; Sellers, J. R. V. Kinetic Prefactors of Reactions on Solid Surfaces. Zeitschrift fur Physikalische Chemie 2013, 227, 1435-1454.

37. Busse, C.; Lazić, P.; Djemour, R.; Coraux, J.; Gerber, T.; Atodiresei, N.; Caciuc, V.; Brako, R.; N’Diaye, A.; Blügel, S.; Zegenhagen, J.; Michely, T. Graphene on $\operatorname{Ir}(111)$ : Physisorption with Chemical Modulation. Phys. Rev. Lett. 2011, 107, 036101-036105.

38. Cavallin, A.; Pozzo, M.; Africh, C.; Baraldi, A.; Vesselli, E.; Dri, C.; Comelli, G.; Larciprete, R.; Lacovig, P.; Lizzit, S.; Alfè, D. Local Electronic Structure and Density of Edge and Facet Atoms at Rh Nanoclusters Self-Assembled on a Graphene Template. ACS Nano 2012, 6, 3034-3043.

39. You, H.; Fain, S. Structure of Carbon Monoxide Monolayers Physisorbed on Graphite. Surf. Sci. 1985, 151, 361-373.

40. Harris, A. B.; Berlinsky, A. J. Mean Field Theory of the Orientational Properties of ( J =1) Hydrogen Molecules on the Surface of Grafoil. Can. J. Phys. 1979, 57, 1852-1869.

41. Alfè, D.; Pozzo, M.; Miniussi, E.; Günther, S.; Lacovig, P.; Lizzit, S.; Larciprete, R.; Burgos, B.; Menteş, T.; Locatelli, A.; Baraldi, A. Fine Tuning of Graphene-Metal Adhesion by Surface Alloying. Sci. Rep. 2013, 3, 2430-2436.

42. Giovannetti, G.; Khomyakov, P.; Brocks, G.; Karpan, V.; Van Den Brink, J.; Kelly, P. Doping Graphene with Metal Contacts. Phys. Rev. Lett. 2008, 101, 026803.

43. Leong, W. S.; Luo, X.; Li, Y.; Khoo, K. H.; Quek, S. Y.; Thong, J. T. L. Low Resistance Metal Contacts to $\mathrm{MoS}_{2}$ Devices with Nickel-Etched-Graphene Electrodes. ACS Nano 2014, 9, 869-877. 
44. Toyoda, K.; Nozawa, K.; Matsukawa, N.; Yoshii, S. Density Functional Theoretical Study of Graphene on Transition-Metal Surfaces: The Role of Metal d-Band in the PotentialEnergy Surface. J. Phys. Chem. C 2013, 117, 8156-8160.

45. Lizzit, S.; Larciprete, R.; Lacovig, P.; Kostov, K. L.; Menzel, D. Ultrafast Charge Transfer at Monolayer Graphene Surfaces with Varied Substrate Coupling. ACS Nano 2013, 7, 4359-4366.

46. Brugger, T.; Günther, S.; Wang, B.; Dil, J.; Bocquet, M.-L.; Osterwalder, J.; Wintterlin, J.; Greber, T. Comparison of Electronic Structure and Template Function of Single-Layer Graphene and a Hexagonal Boron Nitride Nanomesh on Ru(0001). Phys. Rev. B 2009, 79, 045407-045412.

47. Abrami, A.; Barnaba, M.; Battistello, L.; Bianco, A.; Brena, B.; Cautero, G.; Chen, Q. H.; Cocco, D.; Comelli, G.; Contrino, S.; DeBona, F.; Di Fonzo, S.; Fava, C.; Finetti, P.; Furlan, P.; Galimberti, A.; Gambitta, A.; Giuressi, D.; Godnig, R.; Jark, W. et al. Super ESCA: First Beamline Operating at ELETTRA. Rev. Sci. Instrum. 1995, $66,1618-1620$.

48. Schlichting, H.; Menzel, D. Techniques for Attainment, Control, and Calibration of Cryogenic Temperatures at Small Single-Crystal Samples under Ultrahigh Vacuum. Rev. Sci. Instrum. 1993, 64, 2013-2022.

49. Barinov, A.; Malcioglu, O. B.; Fabris, S.; Sun, T.; Gregoratti, L.; Dalmiglio, M.; Kiskinova, M. Initial Stages of Oxidation on Graphitic Surfaces: Photoemission Study and Density Functional Theory Calculations. J. Phys. Chem. C 2009, 113, 9009-9013.

50. Susi, T.; Kaukonen, M.; Havu, P.; Ljungberg, M. P.; Ayala, P.; Kauppinen, E. I. Core Level Binding Energies of Functionalized and Defective Graphene. Beilstein J. Nanotechnol. 2014, 5, 121-132. 
51. Baraldi, A.; Barnaba, M.; Brena, B.; Cocco, D.; Comelli, G.; Lizzit, S.; Paolucci, G.; Rosei, R. Time Resolved Core Level Photoemission Experiments with Synchrotron Radiation. J. Electron Spectrosc. Relat. Phenom. 1995, 76, 145-149.

52. Doniach, S.; Sunjic, M. Many-Electron Singularity in X-Ray Photoemission and X-Ray Line Spectra from Metals. J. Phys. C: Solid State Phys. 1970, 3, 285-291.

53. Shirley, D. High-Resolution X-Ray Photoemission Spectrum of the Valence Bands of Gold. Phys. Rev. B 1972, 5, 4709-4714.

54. Végh, J. The Analytical Form of the Shirley-Type Background. J. Electron Spectrosc. Relat. Phenom. 1988, 46, 411-417.

55. Hohenberg, P.; Kohn, W. Inhomogeneous Electron Gas. Phys. Rev. 1964, 136, B864B871.

56. Kohn, W.; Sham, L. J. Self-Consistent Equations Including Exchange and Correlation Effects. Phys. Rev. 1965, 140, A1133-A1138.

57. Kresse, G.; Furthmüller, J. Efficient Iterative Schemes for Ab Initio Total-Energy Calculations Using a Plane-Wave Basis Set. Phys. Rev. B 1996, 54, 11169-11186.

58. Blöchl, P. E. Projector Augmented-Wave Method. Phys. Rev. B 1994, 50, 17953-17979.

59. Kresse, G.; Joubert, D. From Ultrasoft Pseudopotentials to the Projector AugmentedWave Method. Phys. Rev. B 1999, 59, 1758-1775.

60. Perdew, J. P.; Burke, K.; Ernzerhof, M. Generalized Gradient Approximation Made Simple. Phys. Rev. Lett. 1996, 77, 3865-3868.

61. Zhang, Y.; Yang, W. Comment on "Generalized Gradient Approximation Made Simple". Phys. Rev. Lett. 1998, 80, 890-890. 
62. Grimme, S.; Antony, J.; Ehrlich, S.; Krieg, H. A Consistent and Accurate Ab Initio Parametrization of Density Functional Dispersion Correction (DFT-D) for the 94 Elements H-Pu. J. Chem. Phys. 2010, 132, 154104.

63. Dion, M.; Rydberg, H.; Schröder, E.; Langreth, D. C.; Lundqvist, B. I. Van der Waals Density Functional for General Geometries. Phys. Rev. Lett. 2004, 92, 246401-246405.

64. Román-Pérez, G.; Soler, J. M. Efficient Implementation of a van der Waals Density Functional: Application to Double-Wall Carbon Nanotubes. Phys. Rev. Lett. 2009, 103.

65. Klimeš, J.; Bowler, D. R.; Michaelides, A. Chemical Accuracy for the van der Waals Density Functional. J. Phys.: Condens. Matter 2009, 22, 022201.

66. Hamada, I. Van der Waals Density Functional Made Accurate. Phys. Rev. B 2014, 89, 121103. 


\section{Graphical TOC Entry}

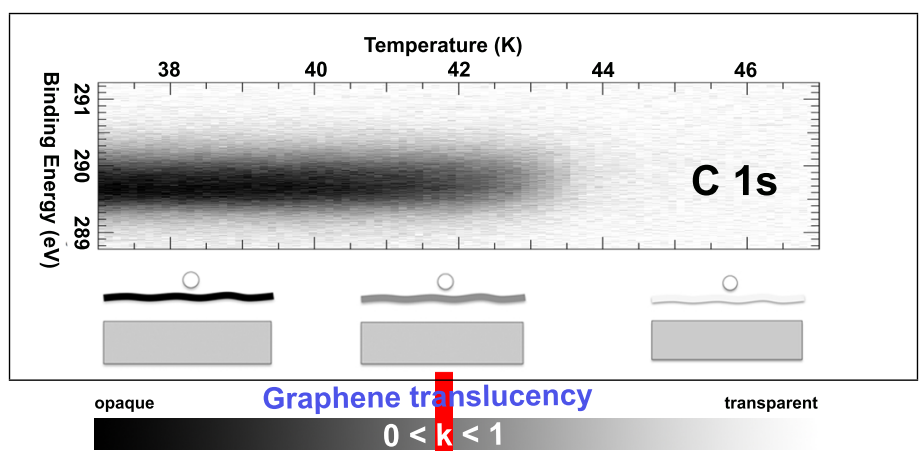

